\title{
Functional Connectivity Among Cognitive Control Networks Moderates the Lagged Association between Sadness and Rumination in Daily Life
}

Lydon-Staley, D.M. ${ }^{1}$, Kuehner, C. ${ }^{2}$, Zamoscik, V. ${ }^{3}$, Huffziger, S. ${ }^{2,4}$, Kirsch, P. ${ }^{3} \&$ Bassett, D.S. ${ }^{1,5,6,7 *}$

${ }^{1}$ Department of Bioengineering, School of Engineering and Applied Sciences, University of Pennsylvania, Philadelphia, PA 19104 USA

${ }^{2}$ Research Group Longitudinal and Intervention Research, Department of Psychiatry and Psychotherapy, Central Institute of Mental Health, Medical Faculty Mannheim, Heidelberg University, 68159 Mannheim Germany

${ }^{3}$ Department of Clinical Psychology, Central Institute of Mental Health, Medical Faculty Mannheim, Heidelberg University, 68159 Mannheim Germany

${ }^{4}$ Institute Psychiatric and Psychosomatics Psychotherapy, Central Institute of Mental Health, Medical Faculty Mannheim, Heidelberg University, 68159 Mannheim Germany

${ }^{5}$ Department of Electrical \& Systems Engineering, School of Engineering and Applied Sciences, University of Pennsylvania, Philadelphia, PA 19104 USA

${ }^{6}$ Department of Neurology, Perelman School of Medicine, University of Pennsylvania, Philadelphia, PA 19104 USA

${ }^{7}$ Department of Physics \& Astronomy, College of Arts and Sciences, University of Pennsylvania, Philadelphia, PA 19104 USA

*Corresponding author:

Danielle S. Bassett, 210 S. 33 ${ }^{\text {rd }}$ Street, 240 Skirkanich Hall, Philadelphia, PA 19104-6321.

Email:dsb@seas.upenn.edu The most recent recent version of this manuscript has been accepted for publication in Translational Psychiatry 


\begin{abstract}
Rumination, the perseverative thinking about one's problems and emotions, is a maladaptive response to sadness and a risk factor for the development and course of depression. A critical challenge hampering attempts to promote more adaptive responses to sadness is that the between-person characteristics associated with the tendency to ruminate following depressed mood remain uncharacterized. We examine the importance of between-person differences in blood-oxygen-level dependent (BOLD) functional networks underlying cognitive control for the moment-to-moment association between sadness and rumination in daily life. We pair functional magnetic resonance imaging with ambulatory assessments measuring momentary sadness and rumination deployed 10 times per day over 4 consecutive days from 58 participants (40 female, mean age $=36.69$ years; 29 remitted from a lifetime episode of Major Depression). Using a multilevel model, we show that rumination increases following increases in sadness for participants with higher than average between-network connectivity of the default mode network and the fronto-parietal network. We also show that rumination increases following increases in sadness for participants with lower than average between-network connectivity of the frontoparietal network and the salience network. In addition, we find that the flexibility of the salience network's pattern of connections with brain regions across time is protective against increases in rumination following sadness. Our findings highlight the importance of the neural correlates of cognitive control for understanding maladaptive responses to sadness and also support the value of large-scale functional connectivity networks for understanding cognitive-affective behaviors as they naturally occur during the course of daily life.
\end{abstract}

Keywords: rumination; depressed mood; experience-sampling; functional connectivity; cognitive control 


\section{INTRODUCTION}

The experience of sadness is often functional as it highlights a discrepancy between one's actual state and one's desired state (Carver \& Scheier, 1998). The cognitive-affective sequelae of sadness, however, do not always support health and well-being. Rumination is a mode of responding to distress or depressed mood that entails perseverative thinking about one's problems or emotions (Nolen-Hoeksema, 1991; Nolen-Hoeksema et al., 2008). While rumination may give individuals the impression that they are solving their problems (Papageorgiou \& Wells, 2001), rumination impairs problem solving and is associated with the endorsement of more negative cognitions (Kuehner et al., 2009), negatively biased interpretations of hypothetical scenarios, and the generation of less effective solutions to problems (Hasegawa et al., 2016), especially for individuals in dysphoric states (Kuehner et al., 2009, Lyubomirsky \& NolenHoeksema, 1995). Evidence that rumination is a maladaptive response to negative mood comes from studies indicating that rumination is a less effective approach to down-regulating negative emotions in daily life in comparison to other emotion regulation strategies (Lennarz et al., 2018), that engaging in rumination when distressed is associated with a greater likelihood of experiencing future depressive episodes (Just \& Alloy, 1997), and that rumination mediates the association between depressed mood and poor self-reported health (Slavish \& Graham-Engeland, 2015).

Although an increased propensity to respond to depressed mood with rumination is a transdiagnostic risk factor for psychopathology from depression (Lyubomirsky et al., 2015, Kuehner, 2017) to multiple other psychopathologies and their co-occurrence (Nolen-Hoeksema \& Watkins, 2011, Kuehner, 2017), much less is known about the between-person differences that moderate the link between sadness and rumination, especially during the course of daily life. The 
extent to which depressed mood is followed by rumination is theorized to be related to impairments in a suite of mental operations referred to as cognitive control (De Raedt \& Koster, 2010; Joormann \& D’Avanzato, 2010; Koster et al., 2011). Cognitive control allows for the flexible allocation of mental resources that underlie the flexible modification of behavior required to achieve one's goals (Braver, 2012; Stokes et al., 2017). From this perspective, the negative elaboration processes characteristic of rumination following the experience of depressed mood or stressful experiences result from difficulties in enacting inhibitory control and diverting attention away from one's moods and emotions. Empirical research supports a role for cognitive control in rumination. Deficits in inhibiting previous mental states are associated with rumination (Whitmer \& Banich, 2007) and cognitive control ability moderates the extent to which negative mood and stressful experiences are followed by rumination (De Lissnyder et al., 2012). Intervention experiments support a causal association between cognitive control and rumination, with cognitive control training reducing the extent to which rumination is deployed in response to low positive affect states in daily life (Hoorelbeke et al., 2016).

Further insight into between-person differences in the association between sadness and rumination may be gained by considering neural correlates of cognitive control. Connectivity among the default mode network, the fronto-parietal network, and the salience network provides an index of cognitive control ability. The default mode network (for review see Buckner et al., 2008) is characterized by a tendency to deactivate during tasks and to activate at rest as well as during self-referential tasks (Mazoyer et al., 2001; Shulman et al., 1997). The fronto-parietal network is comprised of regions that have established roles in sustained attention and working memory (Curtis \& D'Esposito, 2003; Mars \& Grol, 2007), response selection (Jiang \& Kanwisher, 2003), and response suppression (Ridderinkhof et al., 2004), and is essential for 
goal-directed behavior. The salience network facilitates access to cognitive control by balancing activity between the default mode network and the fronto-parietal network (for reviews see Uddin, 2015; Menon, 2015). Evidence supporting the role of the salience network in toggling fronto-parietal network and default mode network activity comes from studies of auditory segmentation, visual oddball attention, and rest that use Granger causality to determine the causal relation among salience network regions (fronto-insular cortex and anterior cingulate cortex) and both fronto-parietal network and default mode network activity (Sridharan et al., 2008).

Intuitively, a brain that has the capacity to respond adaptively to demands allows for greater flexibility in responding to emotional experiences. Indeed, cognitive models emphasize that the modulation and regulation of emotion content and duration is an instance of cognitive control (Ochsner et al., 2012; Zelazo \& Cunningham, 2007). Here, we examine the association between rumination following increases in sadness during daily life and functional connectivity among the default mode network, the fronto-parietal network, and the salience network during sad mood induction. We test the hypothesis that greater propensity to experience rumination in daily life following increases in sadness is associated with patterns of functional connectivity among the default mode network, the fronto-parietal network, and the salience network that are associated with cognitive control. Based on work indicating that the salience network is a uniquely flexible system, exhibiting substantial time-varying functional interactions with other functional networks (Chen et al., 2016), we also test the moderating role of salience network flexibility on the association between sadness and rumination. We show that decreased connectivity between the salience network and the fronto-parietal network, and increased connectivity between the default mode network and the fronto-parietal network, are associated 
with a greater tendency to respond to increases in sadness with rumination. In addition, we show that high salience network flexibility is protective against responding to increases in sadness with rumination. Our findings provide support for theories of the importance of cognitive control for understanding maladaptive responses to depressed mood and also support the value of largescale functional connectivity networks for understanding cognitive-affective behaviors as they naturally occur in daily life.

\section{RESULTS}

To examine the moderating role of functional connectivity among the default mode network, the fronto-parietal network, and the salience network, as well as salience network flexibility, on the association between sadness and rumination, we used ambulatory assessment data as well as blood-oxygen-level-dependent (BOLD) functional magnetic resonance imaging (fMRI) data acquired during sad mood induction. From an initial sample of 60 participants, we analyzed data from a final sample of 58 participants (see Materials and Methods). Tables S1-S2 provide a detailed description of the sample as well as descriptive statistics for the variables used in the analyses.

\section{Functional Connectivity Moderates the Association Between Sadness and Rumination}

We sought to demonstrate that the propensity to follow increases in sadness with increases in rumination in daily life are moderated by between-person differences in patterns of functional connectivity among three brain networks with established roles in cognitive control (for reviews see Uddin, 2015; Menon, 2015). We also examined the importance of salience network flexibility for understanding rumination due to the positive association between salience 
network flexibility and cognitive flexibility (Chen et al., 2016). We present the results of the multilevel model used to test our hypotheses (see Table 1). As shown in Table 1, there is evidence for moderation of the association between previous moment's sadness and rumination for between network connectivity of the default mode network and the fronto-parietal network $(\gamma=11.65, p=0.002)$, for between-network connectivity of the fronto-parietal network and the salience network $(\gamma=-11.15, p<0.001)$, and for salience network flexibility $(\gamma=$ $-10.10, p=0.02$ ). We present the findings associated with all three significant moderating relationships in greater detail below.

[TABLE 1 ABOUT HERE]

\section{Fronto-parietal Network and Salience Network Connectivity}

Based on the role of the salience network in toggling fronto-parietal and default mode network activity to facilitate access to cognitive control resources (Menon \& Uddin, 2010; Sridharan et al., 2008), we hypothesized that stronger connectivity between the fronto-parietal network and the salience network would be associated with a reduced tendency to experience rumination following increases in sadness. In line with this hypothesis, we observe a significant interaction between fronto-parietal network and salience network connectivity and the association between previous moment's sadness and rumination $(\gamma=-11.15, p<0.001)$. We further consider the significant interaction and observe that the regression of rumination on previous moment's sadness is significant and positive for participants with relatively weak connectivity among these networks (Figure 1B). These results indicate that participants with low levels of fronto-parietal network and salience network connectivity experience significant 
increases in rumination following moments of higher than usual sadness (Figure 1C). In contrast, participants with higher levels of fronto-parietal network and salience network connectivity show no significant moment-to-moment association between rumination and sadness.

[FIGURE 1 ABOUT HERE]

\section{Default Mode Network and Fronto-parietal Network Connectivity}

Based on findings that greater strength of connectivity between regions of the default mode network and the fronto-parietal network are associated with poor cognitive performance (Hampson et al., 2010; Kelly et al., 2008), we hypothesized that stronger connectivity between the default mode network and the fronto-parietal network would be associated with a greater tendency to ruminate following increases in sadness. In line with this hypothesis, we observe a significant interaction between default mode network and fronto-parietal network connectivity and the association between previous moment's sadness and rumination $(\gamma=11.65, p=0.002)$. Post-hoc analysis of the significant interaction reveals that the regression of rumination on sadness is significant and positive for participants with relatively high levels of connectivity between these networks (Figure 1E). As shown in Figure 1F, participants with high levels of default mode network and fronto-parietal network connectivity experience significant increases in rumination following moments of higher than usual sadness. In contrast, participants with low levels of default mode network and fronto-parietal network connectivity show no changes in rumination following moments of higher than usual sadness. 


\section{Salience Network Flexibility}

Based on findings that the salience network flexibility is positively associated with cognitive flexibility (Chen et al., 2016), we hypothesized that greater flexibility of the salience network would be protective against the propensity to follow increases in sadness with rumination. In line with this hypothesis, we observe a significant interaction between salience network flexibility and the association between previous moment's sadness and current rumination $(\gamma=-10.10, p=0.02)$. We further consider the significant interaction and observe that the regression of rumination on previous moment's sadness is significant and positive for participants with low values of salience network flexibility (Figure 2E). Participants with low levels of salience network flexibility experience significant increases in rumination following moments of higher than usual sadness (Figure 2F). In contrast, participants with higher levels of salience network flexibility show no significant moment-to-moment association between rumination and sadness. Notably, additional analyses indicate that the moderating effect of network flexibility is specific to the salience network with no evidence for a moderating effect of default mode network or fronto-parietal network flexibility on the association between sadness and rumination emerging (Table S3).

[FIGURE 2 ABOUT HERE]

\section{Additional Sensitivity and Robustness Analyses}

With additional analyses, we examine the extent to which the moderating effects of fronto-parietal network and salience network connectivity, default mode network and frontoparietal network connectivity, and salience network flexibility on the association between 
sadness and rumination are robust to several choices in our pipeline. Specifically, we observe that the effects are robust to (i) the removal of participants with greater than $20 \%$ of volumes with excessive head motion (framewise displacement $>0.5 \mathrm{~mm}$ ) (Table S4), (ii) the removal of participants with movement of greater than $10 \%$ of volumes with excessive head motion (framewise displacement $>0.5 \mathrm{~mm}$ ) (Table S5), (iii) the removal of non-significant covariates (except for non-significant main effects for which interactions are significant) (Table S6), (iv) the inclusion of group status (remitted depressed versus healthy controls) rather than the continuous measure of depressive symptoms (Table S7), and (v) when controlling for previous moment's level of rumination (Table S8).

\section{DISCUSSION}

Rumination is a maladaptive response to sadness. Characterizing between-person differences associated with the propensity to respond to increases in sadness with rumination will facilitate attempts to promote more adaptive responses to sadness. Building off theories and recent empirical investigations highlighting the potential role for individual differences in cognitive control in moderating the tendency to ruminate in response to low mood (De Lissnyder et al., 2012; Koster et al., 2011), we tested the moderating role of functional connectivity among brain networks involved in the successful performance of cognitive control on the association between sadness and rumination (Uddin, 2015). More specifically, we show that between-person differences in functional connectivity among the default mode network, the fronto-parietal network, and the salience network during sad mood moderate the moment-to-moment association between sadness and rumination in daily life. As hypothesized, increased betweennetwork connectivity of the default mode network and the fronto-parietal network, and decreased between-network connectivity of the salience network and the fronto-parietal network 
connectivity, are associated with increases in rumination following increases in sadness. In addition, we observe that participants with flexible engagement of the salience network across time show no tendency to increase rumination following increases in sadness. However, between-network connectivity of the salience network and default mode network does not moderate the association between sadness and rumination.

The finding that patterns of functional connectivity associated with cognitive control moderate the association between sadness and rumination provides support for theories that highlight the importance of cognitive control in inhibiting the negative elaboration processes characteristic of rumination (De Raedt \& Koster, 2010; Joormann \& D’Avanzato, 2010). Individuals with greater cognitive control ability may be better able to inhibit perseverative thinking and to divert attention away from one's negative mood to a greater extent than individuals with lower cognitive control ability. Individuals with poor cognitive control ability, in contrast, may experience greater difficulty in employing cognitive control to use more adaptive emotion regulation strategies in response to increases in sadness (e.g., reappraisal), making them more likely to ruminate.

The pattern of findings for default mode network and fronto-parietal network connectivity, and for fronto-parietal network and salience network connectivity, is in line with previous work indicating that greater strength of connectivity between the default mode network and fronto-parietal network is associated with poorer cognitive control performance (Hampson et al., 2010) and that the salience network plays a causal role in switching between fronto-parietal network and default mode network activity (Sridharan et al., 2008). The specificity of the association for fronto-parietal network and salience network connectivity and not default mode 
network and salience network connectivity, however, indicates more complex interactions among the three systems than is currently appreciated in the broader literature.

A fruitful way forward for understanding the patterns of between-person connectivity among the default mode network, fronto-parietal network, and salience network will be to consider the co-occurrence of patterns of connectivity within individuals via person-centered analysis approaches (Muthén \& Muthén, 2000). The approach taken in the present manuscript identifies the relative contributions of particular patterns of functional connectivity in moderating the association between sadness and rumination on average, across the whole sample. The patterns of connectivity, however, do not occur in isolation. Instead, between-network connectivity among the default mode network, the fronto-parietal network, and the salience network co-occur within individuals to different degrees and in different combinations. Personcentered analyses, such as latent profile analysis, will provide an intuitive approach to modeling this co-occurrence of patterns of functional connectivity among the default mode, fronto-parietal, and salience networks and may provide novel insights into the ways in which patterns of connectivity among these networks interact to confer risk for poor cognitive control and, in turn, poor emotion regulation. Based on current theories, we may expect individuals with relatively high between-network connectivity of the salience network with both the default mode network and the fronto-parietal network as well as relatively low between-network connectivity of the default mode network and the fronto-parietal network to have greater cognitive control abilities than individuals exhibiting other patterns of between-network connectivity.

An additional notable finding is that salience network flexibility was protective against experiencing increases in rumination following increases in sadness. This finding provides further support for the role for cognitive control in understanding maladaptive responses to 
depressed mood. Flexibility captures the extent to which nodes interact with nodes in other communities. Nodes that change communities many times may moderate multiple processes and may be essential for dynamic and adaptive processes (Bassett et al., 2011). Indeed, flexibility of the salience network is associated with cognitive flexibility (Chen et al., 2016). Notably, we find that salience network inflexibility is associated with increases in rumination following sadness above and beyond time-invariant (i.e., static) functional connectivity indices. The finding that a dynamic functional connectivity index provides additional information to static indices is in line with the increasing recognition of the time-varying nature of brain network organization (Calhoun et al., 2014; Preti et al., 2017) and its importance for understanding human mood and cognition (Bassett et al., 2015; Betzel et al., 2016; Braun et al., 2015; Chai et al., 2016; Gerraty et al., 2018; He et al., 2018; Shine et al., 2016). The specificity of salience network flexibility and not flexibility of the default mode network or the fronto-parietal network in explaining increases in rumination provides additional evidence that the salience network is uniquely positioned to facilitate interactions with multiple functional networks in order to support flexible, goal-directed engagement with the environment and may therefore represent a more specific neurobiological signature of resilience (see also Figure S1).

\section{Limitations}

It is important to consider the findings in light of the study's strengths and limitations. The dual-use of fMRI and ambulatory assessment protocols provides a bridge between imaging data and behavior as it occurs in natural contexts, providing evidence for the relevance of laboratory fMRI for real-world behavior (see Trull \& Ebner-Priemer, 2013 and Wilson et al., 2014 for discussion). As well as providing for ecological validity, the collection of data as participants go about their daily lives reduces retrospective biases often introduced in 
questionnaires that ask participants to recall and aggregate information about longer periods of time (e.g., previous 30 days; Schwarz, 2007). In line with a number of definitions of rumination (e.g., Nolen-Hoeksema, 1991), the measure of rumination we used captures perceived uncontrollability over negative thoughts. However, rumination is a multifaceted construct (Smith \& Alloy, 2009) and, thus, the results may not extend to other conceptualizations of rumination. Relatedly, intrinsic connectivity among the default mode network, fronto-parietal network, and salience network provides a general index of cognitive control but the component parts of this umbrella term for a suite of functions involved in the flexible modification of behavior could not be examined. Examining specific components of cognitive control that moderate moment-tomoment associations between sadness and rumination will be important for future work given evidence that distinct cognitive control functions are associated with different forms of rumination (Whitmer \& Banich, 2007).

An additional consideration is our measurement of functional connectivity during sad mood induction. The extent to which patterns of functional connectivity associated with cognitive control more generally versus functional connectivity during sad mood induction specifically drives the present results will require further work. This will be an important avenue for future research given that emotional states modulate cognitive control (Gray, 2001; Mueller, 2011), including in the context of remitted depression (Zamoscik et al., 2014). Repeated measures designs containing both passive resting state and sad mood induction protocols will be required to test the specificity of our observed finding to sad mood induction versus rest conditions. Our sample included a group of remitted depressed individuals and healthy controls due to study aims beyond the scope of the present manuscript. Our findings for the association between patterns of functional connectivity and the association between sadness and rumination 
in daily life remained significant when controlling for depressive symptoms. In additional analyses, three-way interactions predicting rumination with previous moment's sadness, functional connectivity, and depression group status were non-significant (Table S9), providing evidence that the associations hold irrespective of group status.

\section{Conclusions}

In summary, the present study extends previous examinations of the association among depressed mood and rumination by demonstrating that functional connectivity among the default mode network, the fronto-parietal network, and the salience network, as well as salience network flexibility, during sad mood induction moderates the lagged, moment-to-moment association between sadness and rumination in daily life. The findings highlight the utility of brain network indices of cognitive control above and beyond self-report and interviewer-rated depressive symptoms in understanding the cognitive-affective sequelae of sadness. The findings also add to a growing literature highlighting the importance of cognitive control for understanding maladaptive responses to depressed mood through the unique combination of frameworks and methodologies of large-scale functional brain networks and the naturalistic unfolding of cognitive-affective processes in daily life captured via ambulatory assessment.

\section{MATERIALS AND METHODS}

We made use of data from a study designed to provide insight into cognitive and affective function in individuals with remitted major depressive disorder. Detailed information on the larger study is available in Huffziger and colleagues (2013), Timm and colleagues (2017), and Zamoscik and colleagues $(2014,2018)$. Data are available from the corresponding author upon reasonable request.

\section{Participants}


Participants were 29 remitted depressed individuals with $\geq 2$ episodes of major depression disorder and 29 age-, sex-, and education-matched healthy controls without current or lifetime diagnosis for major depressive disorder. Participants were recruited by announcements in local newspapers and on the homepage of the Central Institute of Mental Health (CIMH), Mannheim, Germany. The remitted depressed individuals had to be in partial or full remission, i.e., did not fulfill the criteria of a major depressive episode according to DSM-IV, for at least two months. Exclusion criteria for all participants included clinical diagnoses of bipolar and psychotic disorders, substance dependence, current substance abuse, generalized anxiety disorder, current obsessive-compulsive, post-traumatic stress, and eating disorders according to DSM-IV as well as contraindications for the magnetic resonance imaging. Psychopathologyrelated criteria for inclusion were assessed by a trained clinical psychologist who used the Structural Clinical Interview for DSM-IV axis I (Wittchen et al., 1997) during an individual session.

Sixty participants with complete data were available for use in the present study. A participant with outlier values for the default mode network and fronto-parietal network connectivity variable (9 standard deviations above the mean of this variable) was removed. A second participant with an outlier value for the salience network flexibility $(7$ standard deviations below the mean of this variable) was also removed, leaving data for 58 participants. Detailed information about the sample used for the analyses is presented in Table S1. The study was approved by the local ethics committee of the University of Heidelberg. All participants gave written informed consent after a complete description of the study.

\section{Procedure}


We show a schematic of the procedure in Figure 3. Participants completed a baseline session during which demographic and clinical variables were collected. Following the baseline interview, affective and cognitive state variables were measured by ambulatory assessment (Trull \& Ebner-Priemer, 2013). The ambulatory assessment protocol was completed over four consecutive weekdays with 10 assessments per day using smartphones (HTC Touch Diamond 2) and the software MyExperienceIDE by movisens GmbH (Karlsruhe, Germany). The beginning of each assessment was indicated by a beep, at which point the participants rated momentary mood and cognitive processes. In addition, the ambulatory assessment also included a rumination versus mindful self-focus manipulation. For this manipulation, at the second, third, sixth, eight, and tenth assessment of each day, there was a subsequent 3 minute induction of either ruminative (two induction days) or mindful attention (two induction days) via smartphone screen (randomized cross-over design), followed by another rating of momentary mood and ruminative self-focus. The analyses in the present study focused on pre-induction ratings only (see S1 supplement in Timm et al., 2017).

The fMRI session took place within 2 weeks after the baseline assessment and the ambulatory assessment. Each participant underwent the following four phases: two sad mood inductions (4.5min each), one rumination phase, and one mindfulness phase (both $8 \mathrm{~min})$. Note that the order of the rumination and mindfulness phases was counterbalanced across participants. During the sad mood induction phase, three key words to remind participants of personal negative life events and sad background music (parts of Adagio in G minor by Albinoni) were presented. The key words were presented for 1.5 minutes each during the sad mood induction scan, were chosen by participants, and were related to three negative life events produced after participants were individually assessed immediately prior to the fMRI session. The first sad 
mood induction (before the rumination or mindfulness phase) data was used in the present analyses.

\section{[FIGURE 3 ABOUT HERE]}

\section{Measures}

We made use of demographic and depressive symptom data collected during the baseline session, fMRI data collected during the sad mood induction scan, and sadness and rumination data from the ambulatory assessment.

Momentary Sadness. Sadness was assessed during the ambulatory assessment protocol with the item "At the moment I feel sad", rated on a scale from 0 (not at all) to 6 (very much).

Momentary Rumination. Uncontrollable rumination (e.g., Raes et al., 2008) was assessed during the ambulatory assessment protocol with the item "At the moment, I am stuck on negative thoughts and cannot disengage from them", rated on a scale from 0 ("not at all") to 7 (“very much"). This measure has been used previously in ambulatory assessment studies of rumination (Kuehner et al., 2017, Timm et al., 2018).

Depressive Symptoms. At baseline, depressive symptoms during the previous 2 weeks were assessed with the self-rated Beck Depression Inventory II-Revised (BDI II; Beck et al., 1996; German version: Hautzinger et al., 2006) and the Montgomery and Asberg Depression Rating Scale (MADRS; Montgomery \& Asberg, 1979; German version: Schmidtke et al., 1988) rated by a trained clinical psychologist, both of which have shown good reliability, validity, and sensitivity to symptom changes (Kühner et al., 2007). We created a composite score for depressive symptoms by averaging the z-standardized BDI-II and MADRS scores (Huffziger et al., 2013; Huffziger et al., 2009). 
BOLD Functional Connectivity Networks. A brief summary of our analysis of the functional imaging data is as follows: BOLD time series from the sad mood induction phase were preprocessed and an association matrix representing the functional connectivity among regions of the brain was created before connectivity indices of cognitive control were created. Additional details regarding each of these steps are provided below.

Data Acquisition. One-hundred and eighty T2* weighted EPI images (TR $=1.5 \mathrm{~s}, \alpha=$ $80^{\circ}, \mathrm{TE}=28 \mathrm{~ms}$ ) with 24 slices (slice thickness $4 \mathrm{~mm}$, voxel size $3 \times 3 \times 4 \mathrm{~mm}^{3}$, FOV $192 \mathrm{~mm}$ ) were recorded with a 3 T TIM Trio Scanner with a 12 channel head coil (Siemens Medical Systems, Erlangen, Germany). The first 20 images of each phase were discarded to allow participants to acclimate to the current phase of the experiment.

Data Preprocessing. We processed the fMRI data using tools from FSL (FMRIB's Software Library (www.fmrib.ox.ac.uk.fsl) and AFNI (Cox, 1996) with a preprocessing scheme based on studies that evaluated the performance of a wide variety of preprocessing pipelines in mitigating motion artifact in studies of BOLD functional connectivity (Ciric et al., 2017; LydonStaley et al., 2018). Briefly, time series were processed with a confound regression technique optimized to mitigate the impact of participant motion. Preprocessing included slice time correction, realignment to the median volume, brain extraction, spatial smoothing, and grand mean scaling. Mean white matter and cerebrospinal fluid signals were extracted from the filtered time series using tissue segments generated for each participant. Confound regression included in standard confound signals (six motion parameters plus global signal, white matter, and cerebrospinal fluid) as well as the temporal derivative, quadratic term, and the temporal derivative of the quadratic term (36 parameters in total). We band-bass filtered the functional time series and the confound regressors simultaneously to retain frequencies between 0.01 and 
$0.08 \mathrm{~Hz}$. Despiking was used to smooth outliers in each voxel and high variance confounds were removed (Behzadi, 2007) using nilearn (Abraham et al., 2014).

Creation of an Association Matrix. After preprocessing, we created an association matrix representing the strength of functional connectivity between pairs of brain regions. We defined regions of the salience network, fronto-parietal network, and default mode network based on a commonly applied parcellation scheme (Power et al., 2011) that has been previously used to examine interactions among the default mode network, fronto-parietal network, and salience network (e.g., Chen et al., 2016). For each region, we extracted a time series of the BOLD signal separately for each individual. All regions were modeled as $10 \mathrm{~mm}$ diameter spheres around the center coordinates. The extracted time series were the average time series for all voxels within the sphere. We calculated the wavelet coherence matrix, $C$, using the extracted BOLD time series. Each element of $C_{i j}$ represented the magnitude squared coherence of the scale two (0.0625-0.125 Hz) Daubechies wavelet (length 4) decomposition of the time series of region $i$ and region $j$. We based our choice of frequency on previous work demonstrating sensitivity to neural processes (Bassett et al., 2011; Betzel et al., 2016; Zhang et al., 2016).

Between-Network Connectivity Indices. We labeled regions by the putative functional systems in which they resided. To examine between-network connectivity among the salience network, the default mode network, and the fronto-parietal network, we calculated betweennetwork connectivity as the mean value of the association matrix elements representing functional connections between the regions of the salience network and the regions of the default mode network, between the regions of the salience network and the regions of the fronto-parietal network, and between the regions of the default mode network and the fronto-parietal network. This provided us with three between-system connectivity indices: default mode network and 
salience network connectivity, default mode network and fronto-parietal network connectivity, fronto-parietal network and salience network connectivity.

Flexibility of the Salience Network. In addition to between-network connectivity indices, we created a dynamic functional connectivity index that quantifies the extent to which nodes of the salience network interact with nodes outside of its community (Figure 2A-2D). We used a sliding window and multilayer-modularity maximization approach to create indices of the timevarying interactions of nodes of the network with nodes of other functional networks. To create dynamic brain networks, the time series for each brain region was divided into $T=15$ sliding time windows, each 20 TRs (30 seconds) in duration, with 50\% overlap. The choice of window length was consistent with work indicating that window lengths of 30-60 seconds are reasonable for balancing the capacity to capture dynamics in BOLD signal and the ability to obtain accurate connectivity estimates within windows (Leonardi \& Van De Ville, 2015) (although we acknowledge that shorter windows can also be useful for some questions; Braun et al. 2015, 2016). An added benefit of this choice was that it also allowed us to achieve even window lengths across the experiment. Within each window, edges between all nodes were estimated via wavelet coherence. The result was a time-ordered set of functional connectivity matrices for each subject.

We transformed the time-varying functional connectivity matrices into an ordered set of adjacency matrices (windows 1-15 in the present case), and subsequently into a multilayer network (Bassett et al., 2013; Mucha et al., 2010). In this multilayer network, the graph in one time window is linked to the graph in adjacent time windows via identity edges that connect a node in one time window to the same node in neighboring time windows. Multilayer modularity is defined as 


$$
Q=\frac{1}{2 \mu} \sum_{i j l r}\left\{\left(A_{i j l}-\gamma_{l} P_{i j l}\right) \delta_{l r}+\delta_{i j} \omega_{j l r}\right\} \delta\left(g_{i l}, g_{j r}\right)
$$

where $A_{i j l}$ is the edge weight between node $i$ and node $j$ in time window $l ; P_{i j l}$ is the expected weight of the edge connecting node $i$ and node $j$ under a specified null model; $\gamma_{l}$ is a structural resolution parameter of layer $l$ that tunes the number of communities identified; $g_{i l}$ is the community assignment of node $i$ in layer $l, g_{j r}$ gives the community assignment of node $j$ in layer $r, \delta\left(g_{i l}, g_{j r}\right)=1$ if $g_{i l}=g_{j r}$ and 0 otherwise; the total network edge weight is $\mu=\frac{1}{2} \sum_{j r} \kappa_{j r}$; the strength of node $j$ in layer $l$ is $\kappa_{j l}=\kappa_{j l}+c_{j l}$; the intra-layer strength of node $j$ in layer $l$ is $\kappa_{j l}=\sum_{i} A_{i j l} ;$ and the inter-layer strength of node $j$ in layer $l$ is $c_{j l}=\sum_{r} \omega_{j l r}$.

We implemented multilayer modularity maximization in MATLAB (Jeub et al., 2011) and applied the procedure to each subject's functional connectivity matrices separately. The algorithm was applied with a default structural resolution parameter, $\gamma_{l}$, of 1 and an inter-layer strength parameter, $\omega_{j l r}$, of 1 . As the algorithm is non-deterministic, the algorithm was iterated 100 times for each subject. This procedure resulted in $100 n \times m$ matrices for each participant, where $n$ is the number of nodes (264) and $m$ is the number of sliding windows (i.e., 15), indicating the community allegiance of each node during each sliding window.

We used the matrices indicating community assignment of nodes across sliding windows to create node flexibility indices. Node flexibility captures the number of times a node changes communities across time, normalized by the number of times the node could have changed communities (Bassett et al., 2011). Formally, node flexibility is defined as

$$
f_{i}=\frac{m}{T-1}
$$

where $f_{i}$ is the flexibility of node $i, m$ is the number of times node $i$ changed communities, and $T$ is the number of sliding windows. Using the Network Community Toolbox 
(http://commdetect.weebly.com), node flexibility was calculated for each subject across each pipeline as the average flexibility value across the 100 iterations of the dynamic community detection procedure. Salience network flexibility was calculated as the average of $f_{i}$ over all nodes within the salience network. Previous work shows that the association between network flexibility and cognitive flexibility is specific to the salience network (Chen et al., 2016). For completeness, we also created flexibility indices for the default mode network and the frontoparietal network to use in supplemental specificity analyses (Table S3).

Participant Motion During Sad Mood Induction. We calculated framewise displacement (FD) of the BOLD time series to provide an index of in-scanner motion during the sad mood induction phase (Power et al., 2012). Framewise displacement is defined as the sum of the absolute values of the derivatives of the six realignment parameters created during head movement correction. Two motion-related variables were calculated. The first was the mean FD of the BOLD time series for each participant, which we used as a covariate in analyses. The second was the percent of volumes with excessive motion (FD > 0.05), which we used to test the robustness of the findings to the exclusion of participants with excessive motion.

\section{Data Analysis}

We tested the association among sadness and rumination, as well as the moderating effect of between-system functional connectivity and salience network flexibility during sad mood induction, using a multilevel model (Snijders \& Bosker, 2012). The sadness variable was lagged by one time point to create previous time point $(t-1)$ sadness scores. This process allowed us to measure the temporal precedence required to increase causal inference of the association between previous moment's sadness on current moment's rumination. The first measurement of each day was removed to ensure that the association between the previous moment's sadness and 
the current moment's rumination was approximately equally spaced. Measurement occasions with missing data for sadness and rumination were removed. Of 2088 possible total measurement assessments, 1995 (95.54\%) were available. To examine any bias that might be introduced due to between-person differences in the number of ambulatory assessment time points available, we estimated the correlation coefficients between the number of time points available and the functional connectivity variables. Correlations among number of occasions of ambulatory assessment data available and between network connectivity of the fronto-parietal network and the salience network $(r(56)=-0.05, p=0.72)$, the default mode network and the salience network $(r(56)=0.01, p=0.96)$, the default mode network and the fronto-parietal network $(r(56)=0.04$, $p=0.76)$, and salience network flexibility $(r(56)=-0.20, p=0.14)$ were not significant. In addition, results of the multilevel model were robust to the inclusion of time points of ambulatory assessment data available per participant as a covariate (Table S10).

After preparing the data, we separated the sadness variable into a within-person and between-person variable to allow for both within-person and between-person inferences related to the association between sadness and rumination (Bolger \& Laurenceau, 2013). A timeinvariant, between-person sadness variable (SadnessBW) was calculated as the arithmetic mean across each individual's, grand-mean (mean of total sample) centered repeated measures. Positive values of this variable indicated usual levels of sadness that were higher than the usual levels of sadness for the average participant. A time-varying, moment-level sadness variable (SadnessWN) was calculated as deviations from the between-person means. Positive values of this variable indicated greater sadness than usual at that measurement occasion, while negative values indicated less sadness than usual at that measurement occasion. We constructed the first level of the multilevel model as follows: 
Level 1:

Rumination $_{i t}=\beta_{0 i}+\beta_{1 i}{\text { Sadness } W N_{i, t-1}}+\beta_{2 i}$ Time $_{i t}+e_{i t}$

where Rumination $_{i t}$ is rumination for person $i$ at time $t, \beta_{0 i}$ indicates the average level of rumination for the prototypical individual in the sample, $\beta_{1 i}$ indicates within-person differences in rumination at time $t$ associated with sadness at time $t-1 ; \beta_{2 i}$ indicates the effect of time in the study on rumination; and $e_{i t}$ are time-specific residuals that were allowed to display autocorrelation (AR1).

Person-specific intercepts and associations from the Level 1 model were specified in the second level of the multilevel model, which we constructed as follows:

Level 2:

$\beta_{0 i}=\gamma_{00}+\gamma_{01}$ DefaultModeNetwork - SalienceNetwor $k_{i}+$ $\gamma_{02}$ DefaultModeNetwork - FrontoparietalNetwork $k_{i}+\gamma_{03}$ FrontoparietalNetworkSalienceNetwork $_{i}+\gamma_{04}$ SalienceNetworkFlexibility $_{i}+\gamma_{05}$ Motion $_{i}+\gamma_{06}$ SadnessBW $_{i}+$ $\gamma_{07}$ Age $_{i}+\gamma_{08}$ Sex $_{i}+\gamma_{09}$ Depression $_{i}+u_{0 i}$

$\beta_{1 i}=\gamma_{10}+\gamma_{11}$ DefaultModeNetwork - SalienceNetwork $k_{i}+$ $\gamma_{12}$ DefaultModeNetwork - FrontoparietalNetwork $k_{i}+\gamma_{13}$ FrontoparietalNetwork SalienceNetwork $_{i}+\gamma_{14}$ SalienceNetworkFlexibility $_{i}+u_{1 i}$

$\beta_{2 i}=\gamma_{20}+u_{2 i}$ 
where the $\gamma$ variables are sample-level parameters and the $u$ variables are residual betweenperson differences that may be correlated with one another but are uncorrelated with the variable $e_{i t}$. Parameters $\gamma_{01}$ to $\gamma_{09}$ indicate the effects of person-level default mode network and salience network connectivity, default mode network and fronto-parietal network connectivity, frontoparietal network and salience network connectivity, salience network flexibility, motion, usual sadness, age, sex, and depressive symptoms on rumination. Parameters $\gamma_{11}$ to $\gamma_{14}$ indicate how between-person differences in default mode network and salience network connectivity, default mode network and fronto-parietal network connectivity, fronto-parietal network and salience network connectivity, and salience network flexibility moderated the association between the previous moment's sadness on the current moment's rumination. Our simultaneous estimation of multiple cross-level interaction effects (i.e., level 2 variables moderating level 1 variables) provides a parsimonious test of our hypotheses but also follows best-practice recommendations for estimating cross-level interaction effects using multilevel modeling (Aguinis, Gottfreson, \& Culpepper, 2013).

We fit the model using lme4 in R (Bates et al., 2015) with incomplete data being treated based on an assumption of being missing at random. Significant interactions were followed-up using the Johnson-Neyman technique (Bauer \& Curran, 2005; Johnson \& Neyman, 1936) using software available online (www.quantpsy.org/interact/hlm2.htm). Statistical significance was evaluated at an $\alpha=0.05$. Between-network connectivity of the default mode network and the salience network was not a significant moderator and, thus, was removed from the final model presented in the Results section (but see Table S11 for results when default mode network and salience network connectivity is included). 


\section{Acknowledgements}

CK and PK would like to acknowledge support from the Deutsche Forschungsgemeinschaft (DFG) through grants KU1464/4-1,2 and PK576/12-1,2. DSB and DML would like to acknowledge support from the John D. and Catherine T. MacArthur Foundation, the Alfred P. Sloan Foundation, the Paul G. Allen Foundation, the Army Research Laboratory through contract number W911NF-10-2-0022, the Army Research Office through contract numbers W911NF-14-1-0679 and W911NF-16-1-0474, the National Institute of Health (2-R01-DC009209-11, 1R01HD086888-01, R01-MH107235, R01-MH107703, R01MH109520, 1R01NS099348 and R21-M MH-106799), the Office of Naval Research, and the National Science Foundation (BCS-1441502, CAREER PHY-1554488, BCS-1631550, and CNS1626008). The content is solely the responsibility of the authors and does not necessarily represent the official views of any of the funding agencies. 
Disclosure of Biomedical Financial Interests and Potential Conflicts of Interest

The authors have no biomedical financial interests or conflicts of interest to disclose. 


\section{References}

Abraham, A., Pedregosa, F., Eickenberg, M., Gervais, P., Mueller, A., Kossaifi, J., ... \& Varoquaux, G. (2014). Machine learning for neuroimaging with scikit-learn. Frontiers in Neuroinformatics, 8, 14.

Aguinis, H., Gottfredson, R.K., \& Culpepper, S.A. (2013). Best-practice recommendations for estimating cross-level interaction effects using multilevel modeling. Journal of Management, 39, 1490-1528.

Bassett, D. S., Yang, M., Wymbs, N. F., \& Grafton, S. T. (2015). Learning-induced autonomy of sensorimotor systems. Nature Neuroscience, 18(5), 744-751.

Bassett, D. S., Porter, M. A., Wymbs, N. F., Grafton, S. T., Carlson, J. M., \& Mucha, P. J. (2013). Robust detection of dynamic community structure in networks. Chaos: An Interdisciplinary Journal of Nonlinear Science, 23(1), 013142.

Bassett, D. S., Wymbs, N. F., Porter, M. A., Mucha, P. J., Carlson, J. M., \& Grafton, S. T. (2011). Dynamic reconfiguration of human brain networks during learning. Proceedings of the National Academy of Sciences, 108(18), 7641-7646.

Bates, D., Maechler, M., Bolker, B., \& Walker, S. (2015). Fitting Linear Mixed-Effects Models Using lme4. Journal of Statistical Software, 67(1), 1-48.

Bauer, D.J., Curran, P.J., 2005. Probing interactions in fixed and multilevel regression: inferential and graphical techniques. Multivariate Behavioral Research, 40, 373-400.

Beck, A.T., Steer, R.A., \& Brown, G.K. (1996). Manual for the Beck Depression InventoryII. San Antonio: The Psychological Corporation.

Behzadi, Y., Restom, K., Liau, J., \& Liu, T. T. (2007). A component based noise correction method (CompCor) for BOLD and perfusion based fMRI. Neuroimage, 37(1), 90-101. 
Betzel, R. F., Satterthwaite, T. D., Gold, J. I., \& Bassett, D. S. (2016). A positive mood, a flexible brain. arXiv preprint arXiv:1601.07881.

Bolger, N., \& Laurenceau, J-P. (2013). Intensive longitudinal methods: An introduction to diary and experience sampling research. New York, NY: Guilford Press.

Braver, T. S. (2012). The variable nature of cognitive control: a dual mechanisms framework. Trends in Cognitive Sciences, 16(2), 106-113.

Braun, U., Schäfer, A., Walter, H., Erk, S., Romanczuk-Seiferth, N., Haddad, L., ... \& MeyerLindenberg, A. (2015). Dynamic reconfiguration of frontal brain networks during executive cognition in humans. Proceedings of the National Academy of Sciences, 112(37), 11678-11683.

Buckner, R. L., Andrews-Hanna, J. R., \& Schacter, D. L. (2008). The brain's default network: Anatomy, function, and relevance to disease. Annals of the New York Academy of Science; 1124, 1-38.

Calhoun, V. D., Miller, R., Pearlson, G., \& Adal1, T. (2014). The chronnectome: time-varying connectivity networks as the next frontier in fMRI data discovery. Neuron, 84(2), 262274.

Carver, C.S., \& Scheier, M.F. (1998). On the self-regulation of behavior. New York, NY: Cambridge University Press.

Chai, L. R., Mattar, M. G., Blank, I. A., Fedorenko, E., \& Bassett, D. S. (2016). Functional network dynamics of the language system. Cerebral Cortex, 26(11), 4148-4159.

Chen, T., Cai, W., Ryali, S., Supekar, K., \& Menon, V. (2016). Distinct global brain dynamics and spatiotemporal organization of the salience network. PLoS Biology, 14, e1002469. 
Ciric, R., Wolf, D. H., Power, J. D., Roalf, D. R., Baum, G. L., Ruparel, K., ... \& Gur, R. C. (2017). Benchmarking of participant-level confound regression strategies for the control of motion artifact in studies of functional connectivity. NeuroImage, 154, 174-187.

Curtis, C.E., \& D'Esposito, M. (2008). The inhibition of unwanted actions. In J. Bargh, P. Gollwitzer, \& E. Moresella (Eds.), The Psychology of Action (pp.72-97). New York, NY: Guilford Press.

De Raedt, R., \& Koster, E. H. (2010). Understanding vulnerability for depression from a cognitive neuroscience perspective: A reappraisal of attentional factors and a new conceptual framework. Cognitive, Affective, \& Behavioral Neuroscience, 10(1), 50-70.

De Lissnyder, E., Koster, E. H., Goubert, L., Onraedt, T., Vanderhasselt, M. A., \& De Raedt, R. (2012). Cognitive control moderates the association between stress and rumination. Journal of Behavior Therapy and Experimental Psychiatry, 43(1), 519-525.

Gerraty, R. T., Davidow, J. Y., Foerde, K., Galvan, A., Bassett, D. S., \& Shohamy, D. (2018). Dynamic flexibility in striatal-cortical circuits supports reinforcement learning. Journal of Neuroscience, 2084-17.

Gray, J. R. (2001). Emotional modulation of cognitive control: Approach-withdrawal states double-dissociate spatial from verbal two-back task performance. Journal of Experimental Psychology: General, 130(3), 436.

Hampson, M., Driesen, N., Roth, J. K., Gore, J. C., \& Constable, R. T. (2010). Functional connectivity between task-positive and task-negative brain areas and its relation to working memory performance. Magnetic Resonance Imaging, 28, 1051-1057.

Hasegawa, A., Nishimura, H., Mastuda, Y., Kunisato, Y., Morimoto, H., \& Adachi, M. (2016). Is trait rumination associated with the ability to generate effective problem solving 
strategies? Utilizing two versions of the Means-Ends Problem-Solving Test. Journal of Rational-Emotive \& Cognitive-Behavior Therapy, 34(1), 14-30.

Hautzinger, M., Keller, F., Kuehner, C., \& Beck, A.T. (2006). Das Beck Depressionsinventar II. Deutsche Bearbeitung und Handbuch zum BDI II. Frankfurt a.M.: Harcout Test Services. He, X., Bassett, D. S., Chaitanya, G., Sperling, M. R., Kozlowski, L., \& Tracy, J. I. (2018). Disrupted dynamic network reconfiguration of the language system in temporal lobe epilepsy. Brain, 141(5), 1375-1389.

Hoorelbeke, K., Koster, E. H., Demeyer, I., Loeys, T., \& Vanderhasselt, M. A. (2016). Effects of cognitive control training on the dynamics of (mal) adaptive emotion regulation in daily life. Emotion, 16(7), 945-956.

Huffziger, S., Ebner-Priemer, U., Zamoscik, V., Reinhard, I., Kirsch, P., \& Kuehner, C. (2013). Effects of mood and rumination on cortisol levels in daily life: An ambulatory assessment study in remitted depressed patients and healthy controls. Psychoneuroendocrinology, $38(10), 2258-2267$.

Huffziger, S., Reinhard, I., \& Kuehner, C. (2009). A longitudinal study of rumination and distraction in formerly depressed inpatients and community controls. Journal of Abnormal Psychology, 118(4), 746.

Jeub, L.G.S., Bazzi, M., Jutla, I.S., \& Mucha, P.J. (2011). A generalized Louvain method for community detection implemented in MATLAB. Available at: http://netwiki.amath.unc.edu/GenLouvain/GenLouvain.

Jiang, Y., \& Kanwisher, N. (2003). Common neural substrates for response selection across modalities and mapping paradigms. Journal of Cognitive Neuroscience, 15, 1080-1094. 
Johnson, P.O., Neyman, J., 1936. Tests of certain linear hypotheses and their applications to some educational problems. Statistical Research Memoirs, 1, 57-93.

Joormann, J., \& D'Avanzato, C. (2010). Emotion regulation in depression: Examining the role of cognitive processes: Cognition \& Emotion Lecture at the 2009 ISRE Meeting. Cognition and Emotion, 24(6), 913-939.

Just, N., \& Alloy, L. B. (1997). The response styles theory of depression: tests and an extension of the theory. Journal of Abnormal Psychology, 106(2), 221-229.

Kelly, A. C., Uddin, L. Q., Biswal, B. B., Castellanos, F. X., \& Milham, M. P. (2008). Competition between functional brain networks mediates behavioral variability. NeuroImage, 39, 527-537.

Koster, E. H., De Lissnyder, E., Derakshan, N., \& De Raedt, R. (2011). Understanding depressive rumination from a cognitive science perspective: The impaired disengagement hypothesis. Clinical Psychology Review, 31(1), 138-145.

Kuehner, C. (2017). Why is depression more common among women than among men? The Lancet Psychiatry, 4(2), 146-58. Epub 2016/11/20. doi: 10.1016/s2215-0366(16)30263-2.

Kuehner, C., Huffziger, S., Liebsch, K. (2009). Rumination, distraction, and mindful self-focus: effects on mood, dysfunctional attitudes, and cortisol stress response. Psychological Medicine, 39, 219-228.

Kuehner, C., Welz, A., Reinhard, I., \& Alpers, G. W. (2017). Lab meets real life: A laboratory assessment of spontaneous thought and its ecological validity. PloS one, 12(9), e0184488.

Kühner, C., Bürger, C., Keller, F., \& Hautzinger, M. (2007). Reliabilität und Validität des revidierten Beck-Depressionsinventars (BDI-II). Der Nervenarzt, 78(6), 651-656. 
Lennarz, H. K., Hollenstein, T., Lichtwarck-Aschoff, A., Kuntsche, E., \& Granic, I. (2018). Emotion regulation in action: Use, selection, and success of emotion regulation in adolescents' daily lives. International Journal of Behavioral Development.

Leonardi, N., \& Van De Ville, D. (2015). On spurious and real fluctuations of dynamic functional connectivity during rest. NeuroImage, 104, 430-436.

Lydon-Staley, D. M., Ciric, R., Satterthwaite, T. D., \& Bassett, D. S. (2018). Evaluation of confound regression strategies for the mitigation of motion artifact in studies of dynamic resting state functional connectivity. Retrieved from psyarxiv.com/rw5zt

Lyubomirsky, S., \& Nolen-Hoeksema, S. (1995). Effects of self-focused rumination on negative thinking and interpersonal problem solving. Journal of Personality and Social Psychology, 69(1), 176-190.

Lyubomirsky, S., Layous, K., Chancellor, J.,\& Nelson, S.K. (2015). Thinking about rumination: the scholarly contributions and intellectual legacy of Susan Nolen-Hoeksema. Annual Review of Clinical Psychology, 11, 1-22.

Mars, R. B., \& Grol, M. J. (2007). Dorsolateral prefrontal cortex, working memory, and prospective coding for action. Journal of Neuroscience, 27(8), 1801-1802.

Mazoyer, B., Zago, L., Mellet, E., Bricogne, S., Etard, O., Houdé, O., ... \& Tzourio-Mazoyer, N. (2001). Cortical networks for working memory and executive functions sustain the conscious resting state in man. Brain Research Bulletin, 54, 287-298.

Menon, V. (2015). Salience network. Brain mapping: An Encyclopedic Reference, 2, 597-611.

Menon, V., \& Uddin, L. Q. (2010). Saliency, switching, attention and control: a network model of insula function. Brain Structure and Function, 214, 655-667. 
Montgomery, S.A., Asberg, M. (1979). A new depression scale designed to be sensitive to change. British Journal of Psychiatry, 134, 382-9.

Mucha, P. J., Richardson, T., Macon, K., Porter, M. A., \& Onnela, J. P. (2010). Community structure in time-dependent, multiscale, and multiplex networks. Science, 328(5980), 876-878.

Mueller, S. (2011). The influence of emotion on cognitive control: relevance for development and adolescent psychopathology. Frontiers in Psychology, 2, 327.

Muthén, B., \& Muthén, L. K. (2000). Integrating person-centered and variable-centered analyses: Growth mixture modeling with latent trajectory classes. Alcoholism: Clinical and Experimental Research, 24(6), 882-891.

Nolen-Hoeksema, S., Wisco, B. E., \& Lyubomirsky, S. (2008). Rethinking rumination. Perspectives on Psychological Science, 3(5), 400-424.

Nolen-Hoeksema, S., \& Watkins, E. (2011). A heuristic for developing transdiagnostic models of psychopathology: explaining multifinality and divergent trajectories. Perspectives on Psychological Science, 6(6), 589-609.

Nolen-Hoeksema, S. (1991). Responses to depression and their effects on the duration of depressive episodes. Journal of Abnormal Psychology, 100(4), 569-582.

Ochsner, K. N., Silvers, J. A., \& Buhle, J. T. (2012). Functional imaging studies of emotion regulation: a synthetic review and evolving model of the cognitive control of emotion. Annals of the New York Academy of Sciences, 1251(1), E1-E24.

Papageorgiou, C., \& Wells, A. (2001). Metacognitive beliefs about rumination in recurrent major depression. Cognitive and Behavioral Practice, 8(2), 160-164. 
Power, J. D., Barnes, K. A., Snyder, A. Z., Schlaggar, B. L., \& Petersen, S. E. (2012). Spurious but systematic correlations in functional connectivity MRI networks arise from subject motion. NeuroImage, 59(3), 2142-2154.

Power, J. D., Cohen, A. L., Nelson, S. M., Wig, G. S., Barnes, K. A., Church, J. A., ... \& Petersen, S. E. (2011). Functional network organization of the human brain. Neuron, 72, 665-678.

Preti, M.G., Bolton, T.A.W., \& Van De Ville, D. (2017). The dynamic functional connectome: State-of-the-art and perspectives. NeuroImage, 160, 41-54.

Raes, F., Hermans, D., Williams, J. M. G., Bijttebier, P., \& Eelen, P. (2008). A “Triple W"model of rumination on sadness: Why am I feeling sad, what's the meaning of my sadness, and wish I could stop thinking about my sadness (but I can’t!). Cognitive Therapy and Research, 32(4), 526-541.

Ridderinkhof, K. R., Van Den Wildenberg, W. P., Segalowitz, S. J., \& Carter, C. S. (2004). Neurocognitive mechanisms of cognitive control: the role of prefrontal cortex in action selection, response inhibition, performance monitoring, and reward-based learning. Brain and Cognition, 56, 129-140.

Schmidtke, A., Fleckenstein, P., Moises, W., \& Beckmann, H. (1988). Studies of the reliability and validity of the German version of the Montgomery-Asberg Depression Rating Scale (MADRS). Schweizer Archiv fur Neurologie und Psychiatrie (Zurich, Switzerland: 1985), 139(2), 51-65.

Schwarz, N. (2007). Retrospective and concurrent self-reports: The rationale for real-time data capture. In A. Stone, S. Shiffman, A. Atienza, \& L. Nebeling (Eds.), The science of real- 
time data capture: Self-reports in health research (pp. 11-26). New York, NY: Oxford University Press.

Shine, J. M., Koyejo, O., \& Poldrack, R. A. (2016). Temporal metastates are associated with differential patterns of time-resolved connectivity, network topology, and attention. Proceedings of the National Academy of Sciences, 113(35), 9888-9891.

Shulman, G. L., Fiez, J. A., Corbetta, M., Buckner, R. L., Miezin, F. M., Raichle, M. E., \& Petersen, S. E. (1997). Common blood flow changes across visual tasks: II. Decreases in cerebral cortex. Journal of Cognitive Neuroscience, 9, 648-663.

Slavish, D. C., \& Graham-Engeland, J. E. (2015). Rumination mediates the relationships between depressed mood and both sleep quality and self-reported health in young adults. Journal of Behavioral Medicine, 38(2), 204-213.

Smith, J. M., \& Alloy, L. B. (2009). A roadmap to rumination: A review of the definition, assessment, and conceptualization of this multifaceted construct. Clinical Psychology Review, 29(2), 116-128.

Snijders, T.A.B., \& Bosker, R.J. (2012). Multilevel analysis: an introduction to basic and advanced multilevel modeling (2nd ed.). London, UK: Sage Publishers.

Sridharan, D., Levitin, D. J., \& Menon, V. (2008). A critical role for the right fronto-insular cortex in switching between central-executive and default-mode networks. Proceedings of the National Academy of Sciences, 105, 12569-12574.

Stokes, M.G., Buschman, T.J., \& Miller, E.K. (2017). Dynamic coding for flexible cognitive control. In T. Egner (Ed.), The Wiley Handbook of Cognitive Control (pp. 221-241). Oxford, UK: Wiley-Blackwell. 
Timm, C., Ubl, B., Zamoscik, V., Ebner-Priemer, U., Reinhard, I., Huffziger, S., ... \& Kuehner, C. (2017). Cognitive and affective trait and state factors influencing the long-term symptom course in remitted depressed patients. PloS one, 12(6), e0178759.

Timm, C., Rachota-Ubl, B., Beddig, T., Zamoscik, V., Ebner-Priemer, U., Reinhard, I., Kirsch, P., Kuehner, C. (2018). Mindfulness-based attention training improves cognitive and affective processes in daily life in remitted patients with recurrent depression. A randomized controlled trial. Psychotherapy and Psychosomatics, May 16:1-3. doi: 10.1159/000488862. [Epub ahead of print].

Trull; T.J. \& Ebner-Priemer, U. (2013). Ambulatory Assessment. Annual Review of Clinical Psychology. 2013 ; 9: 151-176. doi:10.1146/annurev-clinpsy-050212-185510.

Uddin, L. Q. (2015). Salience processing and insular cortical function and dysfunction. Nature Reviews Neuroscience, 16, 55-61.

Whitmer, A. J., \& Banich, M. T. (2007). Inhibition versus switching deficits in different forms of rumination. Psychological Science, 18(6), 546-553.

Wilson, S. J., Smyth, J. M., \& MacLean, R. R. (2013). Integrating ecological momentary assessment and functional brain imaging methods: new avenues for studying and treating tobacco dependence. Nicotine \& Tobacco Research, 16, S102-S110.

Wittchen, H. U., Wunderlich, U., Gruschwitz, S., \& Zaudig, M. (1997). SCID: Structured Clinical Interview for DSM-IV Axis I Disorders. Göttingen: Hogrefe.

Zamoscik, V., Huffziger, S., Ebner-Priemer, U., Kuehner, C., \& Kirsch, P. (2014). Increased involvement of the parahippocampal gyri in a sad mood predicts future depressive symptoms. Social Cognitive and Affective Neuroscience, 9(12), 2034-2040. 
Zamoscik, V.E., Schmidt, S.N.L., Gerchen, M.F., Samsouris,C., Timm, C., Kuehner, C., Kirsch, P. (2018). Respiration pattern variability and related default mode network connectivity are altered in remitted depression. Psychological Medicine Jan 16:1-11. doi: 10.1017/S0033291717003890. [Epub ahead of print].

Zelazo, P.D., \& Cunningham, W.A. (2007). Executive function: Mechanisms underlying emotion regulation. In J.J. Gross (Ed.), Handbook of Emotion Regulation (pp. 135-158). New York, NY: Guilford Press.

Zhang, Z., Telesford, Q. K., Giusti, C., Lim, K. O., \& Bassett, D. S. (2016). Choosing wavelet methods, filters, and lengths for functional brain network construction. PloS one, 11(6), $\mathrm{e} 0157243$. 
Table 1.

Results of the Multilevel Model Examining Associations Among Sadness and Rumination and its Moderation by Between-Network Functional Connectivity and Salience Network Flexibility

\begin{tabular}{|c|c|c|c|}
\hline \multicolumn{4}{|l|}{ FIXED EFFECTS } \\
\hline & Estimate & Standard Error & p-value \\
\hline Intercept & $1.13 * * *$ & 0.16 & $<0.001$ \\
\hline Sadness WN & $0.11 * *$ & 0.04 & 0.004 \\
\hline Time & -0.001 & 0.004 & 0.80 \\
\hline DMN-FPN & -0.77 & 11.04 & 0.94 \\
\hline FPN-SAL & -7.66 & 7.75 & 0.33 \\
\hline SAL Flexibility & -7.80 & 13.20 & 0.56 \\
\hline Motion & -0.02 & 0.72 & 0.98 \\
\hline Sadness BW & $0.68 * * *$ & 0.08 & $<0.001$ \\
\hline Age & 0.01 & 0.01 & 0.37 \\
\hline Sex & -0.29 & 0.15 & 0.05 \\
\hline Depressive symptoms & $0.19 *$ & 0.09 & 0.03 \\
\hline DMN-FPN x Sadness WN & $11.65 * *$ & 3.81 & 0.002 \\
\hline FPN-SAL x Sadness WN & $-11.15^{* * *}$ & 2.84 & $<0.001$ \\
\hline SAL Flexibility x Sadness WN & $-10.10^{*}$ & 4.28 & 0.02 \\
\hline \multicolumn{4}{|l|}{ RANDOM EFFECTS } \\
\hline & Estimate & \multicolumn{2}{|c|}{ Confidence Interval } \\
\hline Intercept & 0.48 & \multicolumn{2}{|c|}{$0.38-0.61$} \\
\hline Sadness WN & 0.06 & \multicolumn{2}{|c|}{$0.01-0.27$} \\
\hline Time & 0.03 & \multicolumn{2}{|l|}{$0.02-0.3$} \\
\hline Correlation (Intercept, Sadness WN) & 0.20 & \multicolumn{2}{|l|}{$-0.56-0.78$} \\
\hline Correlation (Intercept, Time) & 0.41 & \multicolumn{2}{|l|}{$0.03-0.69$} \\
\hline Correlation (Sadness WN, Time) & 0.10 & \multicolumn{2}{|l|}{$-0.43-0.57$} \\
\hline AR1 & 0.03 & \multicolumn{2}{|l|}{$-0.02-0.08$} \\
\hline Residual & 1.09 & \multicolumn{2}{|l|}{$1.06-1.3$} \\
\hline \multicolumn{4}{|l|}{ FIT INDICES } \\
\hline AIC & \multicolumn{3}{|l|}{6250.58} \\
\hline $\mathrm{BIC}$ & \multicolumn{3}{|l|}{6373.59} \\
\hline
\end{tabular}

Notes: $* * * p<0.001, * * p<0.01, * p<0.05$. Sadness $\mathrm{WN}=$ within-person deviated version of sadness; Sadness BW = between-person version of sadness; Depressive symptoms = composite score by averaging z-standardized BDI-II and MADRS scores; DMN = default mode network; $\mathrm{FPN}=$ fronto-parietal network; SAL = salience network; AR1 = autocorrelation; AIC = Aikaike information criterion; $\mathrm{BIC}=$ Bayesian information criterion. Continuous predictors were samplemean centered and time was centered at the middle of the ambulatory assessment protocol to facilitate interpretation of the intercept. Sex was specified as a factor with $1=$ male, $2=$ female; $N$ $=1995$ observations nested within 58 participants. 


\section{Figure Captions}

Figure 1. The conditional relation between sadness and rumination as a function of between-person differences in default mode, fronto-parietal, and salience network connectivity.

Panel A highlights the coordinates of regions in the salience network and the fronto-parietal network as defined by Power et al. (2011). Panel B illustrates the values of fronto-parietal network and salience network connectivity (FPN-SAL) at which the association between previous moment's sadness and rumination is significant. The dashed orange vertical line at 0.003 indicates the value of FPN-SAL connectivity at which the effect of previous moment's sadness on rumination becomes non-significant. The upper bound of the region of significance for the FPN-SAL variable (0.02) is also shown. The range of values we observe in the sample is indicated by the horizontal green line at slope of sadness $=0$. Panel $\mathrm{C}$ indicates the effect of previous moment's sadness on rumination at low and high levels of FPN-SAL connectivity. Low and high values of between-person, sample-mean centered FPN-SAL connectivity reflect plus and minus 1 standard deviation about the mean $(-0.01,0.01)$. Values for the previous moment's sadness on the $\mathrm{x}$-axis reflect plus and minus 1 standard deviation about the mean. The slope of the simple regression of rumination on sadness at low levels of FPN-SAL connectivity is significant such that people with higher than average levels of FPN-SAL connectivity experienced significant increases in rumination following higher than usual levels of sadness. The slope of the simple regression at high levels of FPN-SAL connectivity is not significant. Panel D highlights the coordinates of regions in the default mode network and the fronto-parietal network as defined by Power et al. (2011). Panel E illustrates the values of default mode network and fronto-parietal network connectivity (DMN-FPN) at which the association between previous 
moment's sadness and rumination is significant. The dashed orange vertical line at -0.002 indicates the lower bound of the region of significance on the DMN-FPN connectivity variable at which point the effect of previous moment's sadness on rumination becomes significant. The upper bound is beyond the range of observed data and is not shown. The range of values observed in the sample is indicated by the horizontal green line at sadness $=0$. Panel $\mathrm{F}$ indicates the association between previous moment's sadness and rumination at low and high levels of DMN-FPN connectivity. Low and high values of between-person, sample-mean centered DMNFPN connectivity are defined as plus and minus 1 standard deviation about the mean $(-0.01$, 0.01). Values for the previous moment's sadness on the $\mathrm{x}$-axis reflect plus and minus 1 standard deviation about the mean. The slope of the simple regression at high levels of DMN-FPN connectivity is significant such that people with higher than average levels of DMN-FPN connectivity experienced significant increases in rumination following higher than usual levels of sadness at the previous measurement occasion. The slope of the simple regression of rumination on sadness is not significant at low levels of DMN-FPN connectivity.

Figure 2. The conditional relation between sadness and rumination as a function of between-person differences in salience network flexibility. Panel A illustrates time courses of blood oxygen level-dependent (BOLD) signals from 1 of the 264 brain regions defined by Power et al. (2011). Functional connectivity between the time courses of each pair of brain regions is completed via wavelet coherence at overlapping time windows of 20 TRs in length. Panel B illustrates connectivity matrices indicating the functional connectivity among each pair of brain regions. Four of the 15 sliding windows are illustrated. Panel C illustrates the construction of node flexibility. Multilayer modularity maximization is used to assign each brain region (node; 
$\mathrm{y}$-axis) to a community (assignment indicated by color) at each sliding window ( $\mathrm{x}$-axis). Panel C highlights the coordinates of regions in the salience network as defined by Power et al. (2011). Flexibility of an individual node captures the number of times the node changed community normalized by the number of times the node could have changed communities.

Panel E illustrates the values of salience network flexibility at which the association between previous moment's sadness and rumination is significant. The dashed orange vertical line at 0.005 indicates the value of salience network flexibility at which the effect of previous moment's sadness on rumination becomes non-significant. The upper bound of the region of significance for the salience network flexibility variable (0.04) is not shown as values below this bound are not observed in the sample. The range of values we observe in the sample is indicated by the horizontal green line at previous moment's sadness $=0$. Panel F indicates the effect of previous moment's sadness on rumination at low and high levels of salience network flexibility. Low and high values of between-person, sample-mean centered salience network flexibility reflect plus and minus 1 standard deviation about the mean $(-0.03,0.03)$. Values for the previous moment's sadness on the $\mathrm{x}$-axis reflect plus and minus 1 standard deviation about the mean. The slope of the simple regression of rumination on sadness at low levels of salience network flexibility is significant such that people with lower than average levels of salience network flexibility experienced significant increases in rumination following higher than usual levels of sadness at the previous measurement occasion. The slope of the simple regression at high levels of salience network flexibility is not significant.

Figure 3. Schematic of Study protocol. Participants first completed a baseline session during which demographic and clinical information was collected. Participants then underwent an 
ambulatory assessment protocol. The protocol was completed over four consecutive weekdays with 10 assessments per day. The beginning of each assessment was indicated by a beep, at which point participants rated momentary mood and cognitive processes. Within 2-weeks of the baseline assessment and the ambulatory assessment, each participant underwent an fMRI session. The fMRI session included a sad mood induction. During the sad mood induction, three key words to remind participants of personal negative life events were presented for 1.5 minutes each combined with sad background music (parts of Adagio in G minor by Albinoni). 


\section{Figure 1}

A

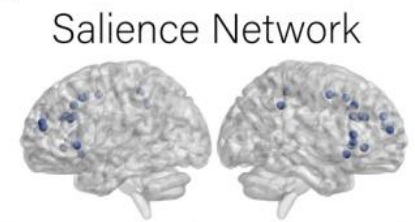

Fronto-parietal Network

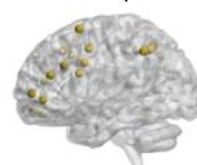

D
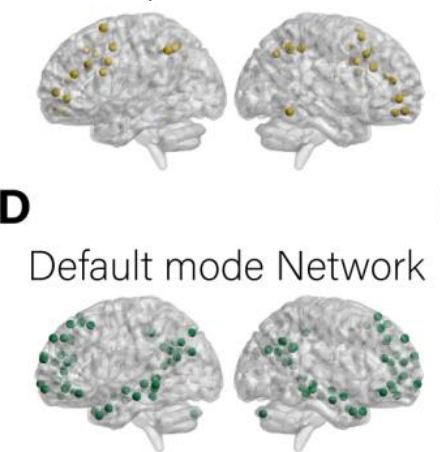

Fronto-parietal Network

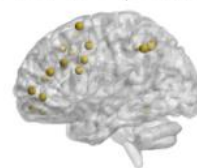

B

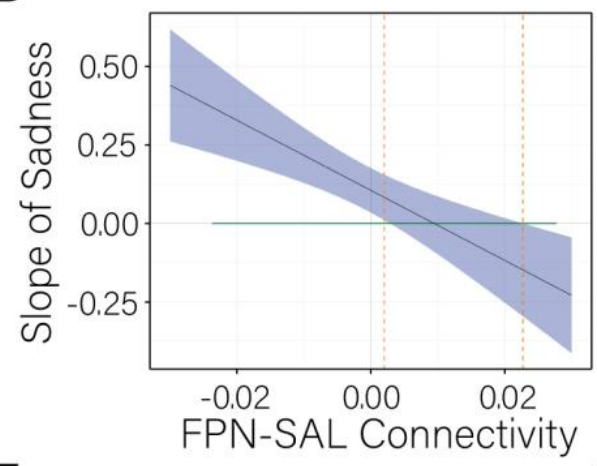

E

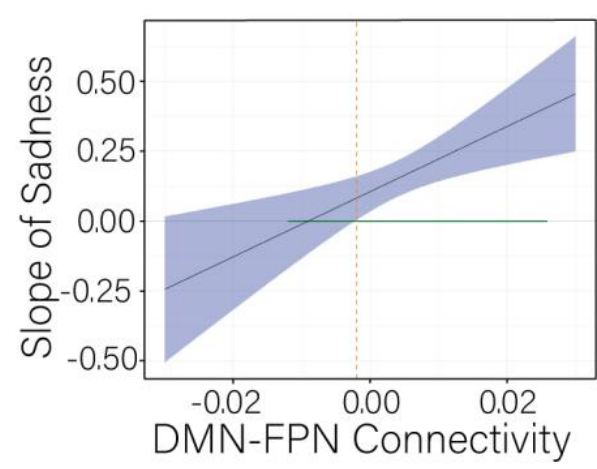

C

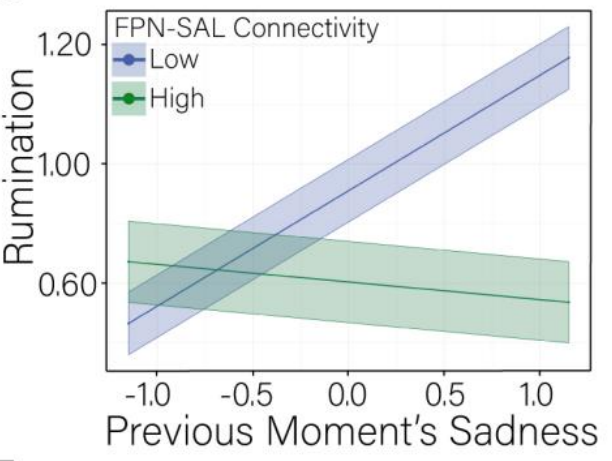

F

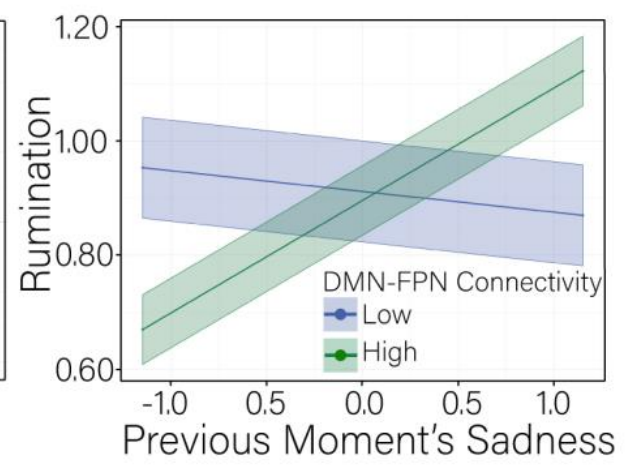




\section{Figure 2}

A

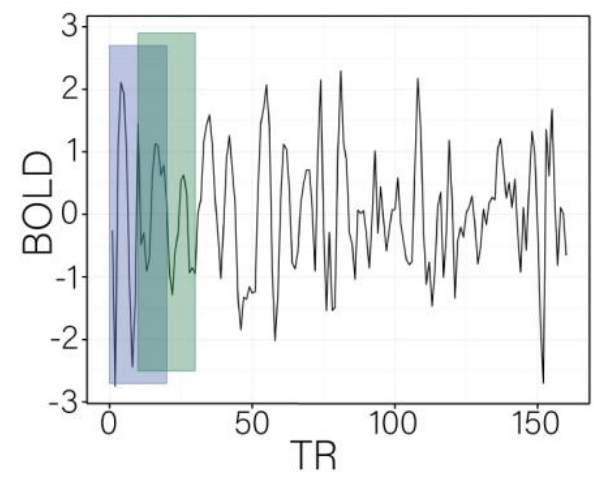

B

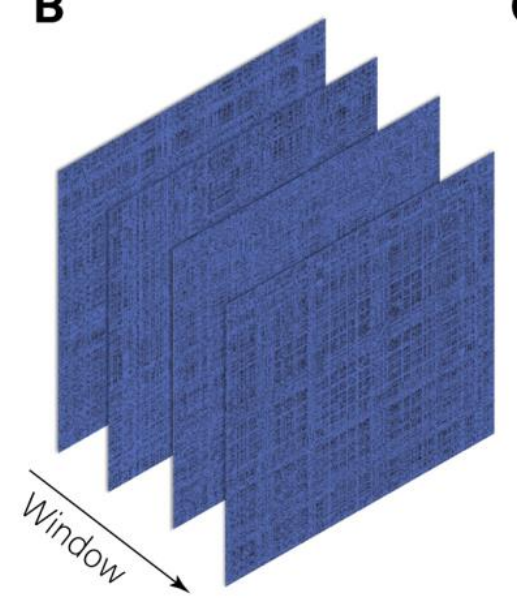

C

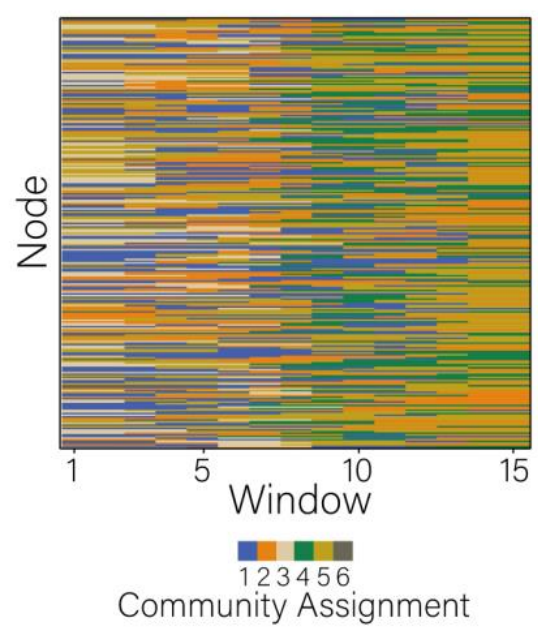

F

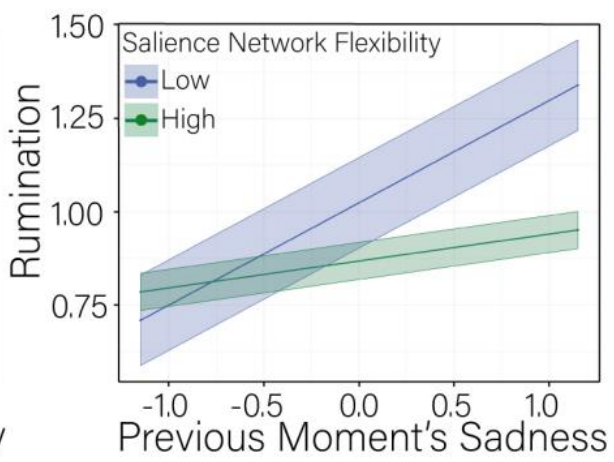

D

Salience Network

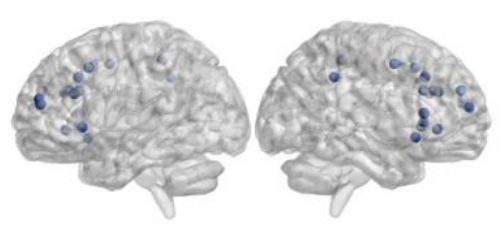

Flexibility $=m / T-1$
E

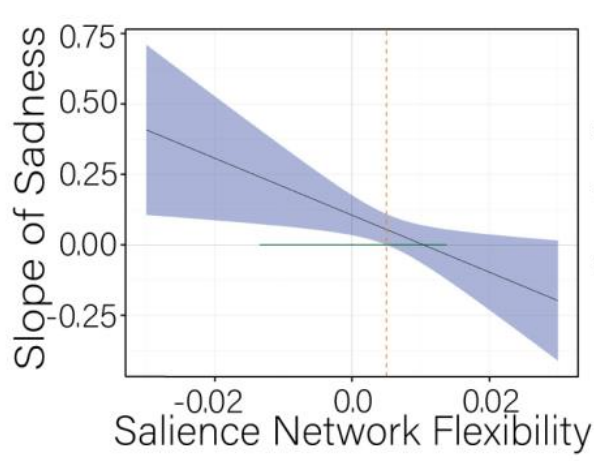




\section{Figure 3}

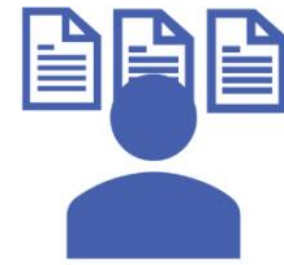

Baseline

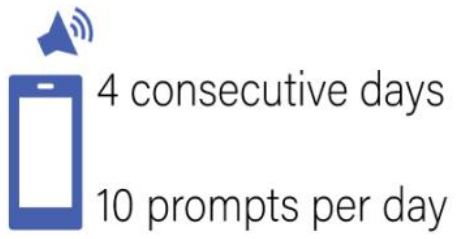

Ambulatory Assessment
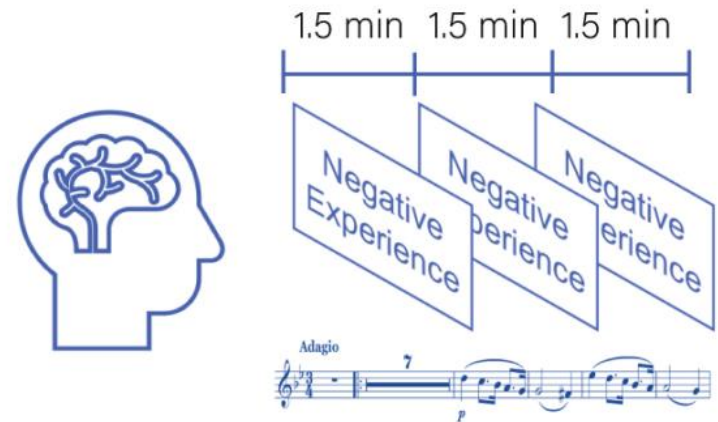

fMRI Sad Mood Induction 